\title{
Phase diagram of a square-well model in two dimensions
}

\author{
Julio C. Armas-Pérez, ${ }^{1}$ Jacqueline Quintana- $\left.\mathrm{H},{ }^{1}, \mathrm{a}\right)$ Gustavo A. Chapela, ${ }^{2}$ \\ Enrique Velasco, ${ }^{3}$ and Guillermo Navascués ${ }^{3}$ \\ ${ }^{1}$ Instituto de Química, Universidad Nacional Autónoma de México, Apdo. Postal 70213, 04510 Coyoacán, \\ México D.F., Mexico \\ ${ }^{2}$ Departamento de Física, Universidad Autónoma Metropolitana-Iztapalapa, Av. San Rafael Atlixco 186, \\ Col. Vicentina, 09340 México D.F., Mexico \\ ${ }^{3}$ Departmento de Física Teórica de la Materia Condensada and Instituto de Física de la Materia Condensada, \\ Facultad de Ciencias M05-605, Universidad Autónoma de Madrid, 28049 Madrid, Spain
}

(Received 11 November 2013; accepted 16 January 2014; published online 11 February 2014)

\begin{abstract}
The phase behavior of a two-dimensional square-well model of width $1.5 \sigma$, with emphasis on the low-temperature and/or high-density region, is studied using Monte Carlo simulation in the canonical and isothermal-isobaric ensembles, and discontinuous molecular-dynamics simulation in the canonical ensemble. Several properties, such as equations of state, Binder cumulant, order parameters, and correlation functions, were computed. Numerical evidence for vapor, liquid, hexatic, and triangular solid is given, and, in addition, a non-compact solid with square-lattice symmetry is obtained. The global phase diagram is traced out in detail (or sketched approximately whenever only inaccurate information could be obtained). The solid region of the phase diagram is explained using a simple mean-field model. (C) 2014 AIP Publishing LLC. [http://dx.doi.org/10.1063/1.4863993]
\end{abstract}

\section{INTRODUCTION}

The square-well (SW) potential continues to be used as a model to explain the phase behaviour of materials. ${ }^{1-4}$ The potential has been used to represent effective interactions in a variety of physical systems such as colloids, proteins, molecular liquids, glasses, and alloys. Variations of it have also been used to analyse more complex liquids exhibiting thermodynamic anomalies such as water, where two typical distances arise due to the orientational molecular interactions. ${ }^{5-10}$

Normal atomic or molecular systems can be modelled with values of the well width $\lambda$ of the order of the diameter of the hard sphere, $\sigma$. Very short-ranged SW models, used for colloidal systems, have been shown to give rise to solidsolid transitions between fcc (compact) structures terminating at critical points due to the "condensation" of neighbours within the first correlation shell inside the well. ${ }^{11}$ At the same time the liquid-vapor coexistence disappears for very shortranged potentials due to the lack of attractive energy for low to moderate densities. The SW model contains more interesting physics related to the possibility that unusual, non-compact, structures can be stabilised in the solid region of the phase diagram when the range is longer. The mechanism is also related to a "condensation" of correlation shells farther than the first. Some time ago Young and Alder ${ }^{12}$ showed that the hexagonal close-packed (hcp) structure may also be stable for $\lambda=1.63 \sigma$ and that, even a non-compact structure such as the body-centered cubic (bcc) could be stable for $\lambda=1.15 \sigma$. Further studies have confirmed these results and widened the set of possible non-compact structures. ${ }^{13}$ Solid-solid transitions between compact (triangular) lattices have been shown

\footnotetext{
a) Author to whom correspondence should be addressed. Electronic mail: jaq@unam.mx
}

to exist also in the two-dimensional (2D) SW model when the range of the well is very short. ${ }^{11}$ The mechanism is the same: nearest-neighbours condensate inside the well to lower the internal energy in order to compensate for the decrease of entropy associated with a larger density. There is no reason to expect that in $2 \mathrm{D}$ larger values of $\lambda$ will not stabilise non-compact structures such as the square lattice.

In addition, it is well known that dimensionality greatly affects the phase behavior and genuine phenomena may appear in 2D. Several cases can be mentioned, one being the Kosterlitz-Thouless-Halpering-Nelson-Young (KTHNY) ${ }^{14,15}$ phase transition in some 2D systems, the effect that confinement has on chiral segregation, ${ }^{16}$ and more recently the existence of 2D carbon (graphene) with several and striking characteristics. ${ }^{17}$ Some other issues like $2 \mathrm{D}$ melting and the hexatic phase, have been studied for a long time, even recently. However, no general consensus has been attained.

A considerable larger number of models have been investigated in $3 \mathrm{D}^{18-29}$ compared to the $2 \mathrm{D}$ case. There are some early simulation studies ${ }^{30}$ to understand the clustering and nucleation in the vapor-liquid transition, a calculation of the critical point, ${ }^{31}$ grand canonical Monte Carlo (GCMC) simulations ${ }^{32}$ for the liquid-vapor interphase. Other works report the vapor-liquid coexistence in a quasi 2D Stockmayer potential ${ }^{33}$ and the nucleation of a 2D Lennard-Jones potential. ${ }^{34}$ The study of solid-solid transitions was extended to Yukawa potentials. ${ }^{11}$ In the present paper, we will focus on a $2 \mathrm{D} \mathrm{SW}$ model with a wide potential well, $\lambda=1.5 \sigma$. Aside from the transitions in the solid region mentioned above, we would like to locate the hexatic phase of the model in relation with the liquid and solid phases at temperatures close to the triple point. A region of hexatic stability has been shown to exist in the neighbourhood of the solid-solid critical point when $\lambda$ is small. ${ }^{35}$ This is not surprising, considering that 
the hard disc model ( $\mathrm{SW}$ when $T \rightarrow \infty$ ) exhibits a hexatic phase that mediates the fluid-solid transition, an otherwise weak transition in that model. The hexatic phase is expected to persist down to low temperatures but, at the same time, the liquid-solid transition will become stronger and it is interesting to understand the role of the hexatic phase in this region of the phase diagram.

In Sec. II we define the particle model and the simulation method in detail. Results are presented in Sec. III, and a short discussion and the conclusions are given in Sec. IV.

\section{MODEL AND METHODS}

The potential model is the standard SW model:

$$
\phi_{S W}(r)= \begin{cases}\infty, & r<\sigma, \\ -\epsilon_{0}, & \sigma<r<\lambda, \\ 0, & r>\lambda .\end{cases}
$$

with $\sigma$ the diameter of the discs and $\lambda$ the range of the potential. In our calculations we take $\lambda=1.5 \sigma$. In the following we will use $\sigma$ as length unit and $\epsilon_{0}$ as energy unit. Therefore, the reduced density $\rho^{*}$ will be defined as $\rho^{*}=\rho \sigma^{2}$, with $\rho$ $=N / A$ and $N$ the number of particles and $A$ the total system area. The reduced temperature will be $T^{*}=k T / \epsilon_{0}$ with $T$ the temperature and $k$ Boltzmann constant. The reduced pressure $p^{*}$ will be defined as $p^{*}=p \sigma^{2} / \epsilon_{0}$.

A theoretical approach, to be explained in Sec. III A, was used to explore the possible stable phases in the solid region. A more serious approach, based on computer simulation, was then undertaken. Two simulation methods and different ensembles were used to explore the phase behaviour: The Monte Carlo (MC) method in the canonical ensemble (NVT) ${ }^{36}$ and in the isothermal-isobaric ensemble (NpT), ${ }^{37}$ and discontinuous molecular dynamics (DMD) ${ }^{38-40}$ in the NVT ensemble. Also, MC simulations were used to obtain the free energy in the solid region.

The number of MC steps for the canonical simulations was $4 \times 10^{6}$ to equilibrate while for production $8 \times 10^{6}$ steps were used. The NpT ensemble simulations were performed using cycles, where one cycle is defined as follows: $4 \mathrm{~N}$ particle moves within the box, and one change of the box area every $4 N$ particle moves.

Several size systems were studied, namely, $N=20^{2}, 32^{2}$, $64^{2}, 128^{2}$, and $256^{2}$. The larger values of $N$ were used to check some of the conclusions. Different box shapes going from square to rectangular were used to simulate dense structures. The ratio of the box lengths, defined as $r_{b o x}=L_{x} / L_{y}$, was adjusted to favor a particular solid structure. For example, in the case of square and triangular structures, the ad hoc box shapes have $r_{b o x}=1$ and $r_{b o x}=2 / \sqrt{3}$, respectively. In the present study other aspect ratios will be used, depending on the case. Moves in the canonical ensemble were accepted with a probability of $30 \%$. In the isothermal-isobaric ensemble $N p T$, fluctuations in the box area were implemented in two different ways: The standard one where both sides are changed simultaneously, and a second, where one of the box sides is chosen at random and its length changed; the latter choice was made to study the structure of solids.
The DMD method ${ }^{38-40}$ can be described as follows: Once the initial positions (triangular network) and velocities are assigned, collision times for each pair of atoms in the system are calculated using an analytical expression. Particles are displaced at constant velocity for a time equal to the minimum collision time and the collision is resolved. New velocities are obtained for the two particles colliding and new possible collisions are calculated for these two particles. The minimum time is calculated again and the cycle is continued until sufficient collisions have been performed. Time saving schemes are applied, such as a collision table with its corresponding maximum collision time, in order to speed up the simulation. The temperature is kept constant by scaling the velocities every 100 collisions to perform the simulation in the NVT ensemble.

\section{A. Order parameters and correlation functions}

To investigate correlation properties in the dense regions, different properties were computed, i.e., different order parameters and their correlations. The local translational order parameter is defined as

$$
\Psi_{T}=\left\langle\frac{1}{N} \sum_{j=1}^{N} e^{i \vec{G} \cdot \vec{r}_{j}}\right\rangle,
$$

where brackets indicate a configurational average. Here $\vec{G}$ is a reciprocal lattice vectors ${ }^{41}$ and $\vec{r}_{j}$ is the vector position of the $j$ th particle. The structure of the solid is captured through the set of reciprocal vectors. For example, for a triangular lattice, $\vec{G}_{\triangle}=\left(2 \pi / a_{\triangle}, 2 \pi / a_{\triangle} \sqrt{3}\right)$, the lattice parameter being given by $a_{\triangle}=\left(2 / \rho^{*} \sqrt{3}\right)^{1 / 2}$. For the square lattice $\vec{G}(2 \pi / a, 0)$ and $a_{\triangle}=\sqrt{\rho^{*-1}}$. Therefore, for a phase having mostly one particular structure, the value of this order parameter is high.

Because the value of $\Psi_{T}$ depends strongly on the direction of the reciprocal lattice vectors, it was necessary to choose the angle that maximized the translational order parameter value. Therefore, $\Psi_{T}$ was calculated for angles between 0 and $2 \pi / 3$, and we took the angle that yielded the highest value of $\Psi_{T}$. The translational correlation function $G_{T}\left(\mathbf{r}-\mathbf{r}^{\prime}\right)=\left\langle\rho^{*}(\mathbf{r}) \rho\left(\mathbf{r}^{\prime}\right)\right\rangle$ associated to the translational order parameter was also calculated in the usual way (see, e.g., Ref. 42). This property provides a precise definition of the translational decay order of the phase.

To characterize the hexatic phase, a bond orientational order parameter was calculated from

$$
\Psi_{6}=\left\langle\frac{1}{N} \sum_{j=1}^{N} \frac{1}{N_{b}} \sum_{k=1}^{N_{b}} e^{i 6 \theta_{k j}}\right\rangle,
$$

where $N_{b}$ is the number of neighbours defined according to the triangulation criterion of Delaunay, ${ }^{43} \theta_{k j}$ is the relative angle formed between the $j$ th particle and the $i$ th neighbor, taking a fixed reference axis. This provides a measure of the alignment of the center of mass position vectors connecting nearest neighbors. ${ }^{41}$ In the hexatic phase this order parameter reaches high values. Similar to the previous case of translational correlation function, the kind of decay of this property is computed via the bond orientational correlation 
function $G_{6}\left(\mathbf{r}-\mathbf{r}^{\prime}\right)$ which is defined in the usual way (see, e.g., Ref. 42) as

$$
G_{6}\left(\mathbf{r}-\mathbf{r}^{\prime}\right)=\left\langle\psi_{6}^{*}(\mathbf{r}) \psi_{6}\left(\mathbf{r}^{\prime}\right)\right\rangle,
$$

where $\psi_{6}(\mathbf{r})$ is defined as the local bond orientational order and it is obtained as

$$
\psi_{6}=\frac{1}{N_{b}} \sum_{k=1}^{N_{b}} e^{i 6 \theta_{k}} .
$$

Assuming that the liquid-hexatic phase transition is described by the KTHNY theory, the transition occurs when the decay exponent of $G_{6}$ is $\eta=-1 / 4$; if $\eta<-1 / 4$ one can say that the system is in the liquid phase. With the aim to verify the order of the transition, system-size analysis was done by calculating the Binder cumulant, ${ }^{44}$ defined as

$$
U_{L}^{4}=1-\frac{\left\langle\Psi_{6}^{4}\right\rangle_{L}}{3\left\langle\Psi_{6}^{2}\right\rangle_{L}^{2}},
$$

where $\Psi_{6}^{2}$ and $\Psi_{6}^{4}$ are the second and fourth moments of the $\Psi_{6}$ distribution.

According to Weber et al., ${ }^{44}$ we can find two scenarios. The phase transition is of first order when the behavior of the cumulant as a function of temperature $T^{*}$ (in case the transition is temperature-driven) for different system sizes is such that all curves intersect at a particular value of $T^{*}$. In the second scenario, we can have a continuous transition. Consistent with the KTHNY theory, the hexatic phase would be defined as an extended critical phase, equivalent to a line of Binder cumulant intersections.

\section{RESULTS}

In this section we focus the discussion separately on the solid-solid and liquid-solid phase-transition boundaries.

\section{A. Solid-solid phase boundaries}

Since the SW width is quite long, more than one correlation shell of neighbours might be inside the well, and one has to carefully analyse possible solid structures that might be stable at low temperatures. Strictly at $T=0$ (where the entropy plays no role), the free energy $F$ is given by the potential energy $E^{(0)}(\rho)$, which in our case is proportional to the number of neighbours inside the well, $N_{n}(\rho)$, i.e., $F(\rho) / N \epsilon_{0}$ $=E^{(0)}(\rho) / N \epsilon_{0}=-N_{n}(\rho) / 2$. After sampling all possible Bravais lattices, we identified two possible periodic solid structures, with two different lattices, in this model. One is the triangular $(\triangle)$ structure, based on a triangular lattice with each site having $N_{n}(\rho)=6$ nearest-neighbour sites. The other is the square $(\square)$ solid, where sites are arranged into a square lattice with $N_{n}(\rho)=4$ or 8 nearest neighbours, depending on the density. That these two structures must necessarily be stable and coexist in some density and temperature range can be understood from the calculation of free energies as a function of density at $T=0$ and for the two structures, Fig. 1. A simple Maxwell construction on these free energies (dashed curves in the figure) shows that the $\square$ solid is stable in the density range $\rho^{*} \in[8 / 9,1]$. The $\triangle$ phase is stable only at its close-packing
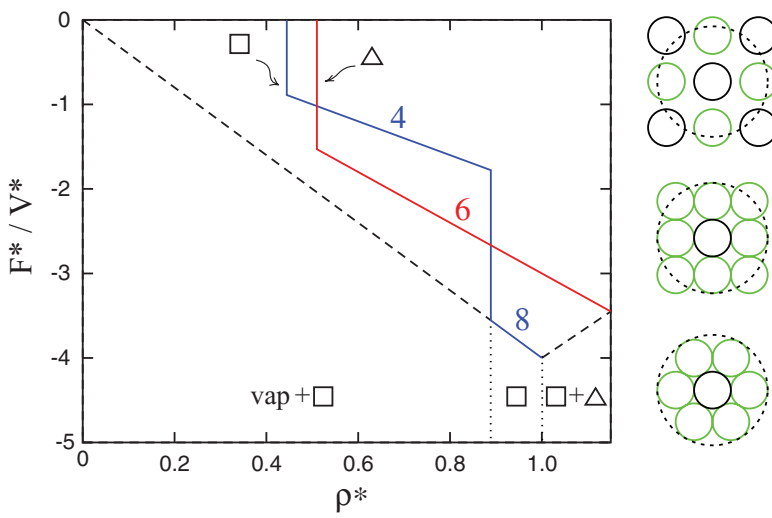

FIG. 1. Left panel: Reduced free energy density $F^{*} / V^{*}$ as a function of reduced density $\rho^{*}$ for the $\square$ solid (blue curve) and the $\Delta$ solid (red curve). The dashed lines indicate the equilibrium free energy density of the system. Dotted vertical lines indicate the density boundaries where the solid is stable. Numbers on the curves indicate the number of nearest neighbours. The density intervals for the vapor and coexistences are indicated by symbols. Right panel: (a) structure with a density such that only four neighbours lie inside the potential well with $\lambda^{*}=1.5$ (indicated by the dashed circle); (b) structure at high (close-packing) density such that eight neighbours lie inside the potential well; and (c) $\triangle$ lattice, with only six neighbours at all densities up to close packing.

density, $\rho^{*}=2 / \sqrt{3} \simeq 1.15$. Two coexistence regions, vapor $+\square$ and $\square+\triangle$, can be identified. In a sample with average density $\rho^{*}<8 / 9$, there is coexistence between a $\square$ solid of density $\rho^{*}=8 / 9$ and a vacuum (vapor). On the other side, the close-packed $\square$ solid coexists with the close-packed $\triangle$ solid. Clearly, it is the fact that, beyond some density value, the second nearest neighbours of the square lattice can go inside the potential well (putting a total of 8 neighbours within the potential range instead of the usual 4) that stabilises the $\square$ solid against the $\triangle$ solid, which can only have 6 neighbours. When $T^{*}>0$ we expect the stability island of the $\square$ phase to continue, at least in some finite range, as well as the $\square$-vapor and $\square-\triangle$ coexisting phases. For detailed calculations, we must resort to simulation or theoretical treatment, to which we now turn.

We have used a simple theory to explore the solid region of the phase diagram at $T^{*}>0$. We write a free energy $F(\rho)$ $=E^{(0)}(\rho)-T S_{\mathrm{HD}}(\rho)$, where the entropy is approximated by $S_{\mathrm{HD}}(\rho)=N k_{b} \log v(\rho)$, the free-volume expression for the hard-disc solid, with $\mathrm{N}$ the number of particles, $k_{b}$ the Boltzmann factor, and $v(\rho)$ is the exact free volume of the hard disc solid which, in turn, can be very accurately approximated in the manner proposed in Velasco et al. ${ }^{45}$ i.e.,

$$
v(\rho)=\frac{1}{\rho}\left[\frac{a-\sigma}{a / 2}\right]^{2} .
$$

Since most neighbours of a given particle will be almost inside the potential well, the effect of temperature on internal energy is not expected to be large. However, due to the SW attraction, particles will be less localized and therefore $S_{\mathrm{HD}}(\rho)$ should underestimate the actual SW entropy. The free energies of the two solids, $\square$ and $\triangle$, were calculated as a function of density, and from them pressures and chemical potentials were obtained. 


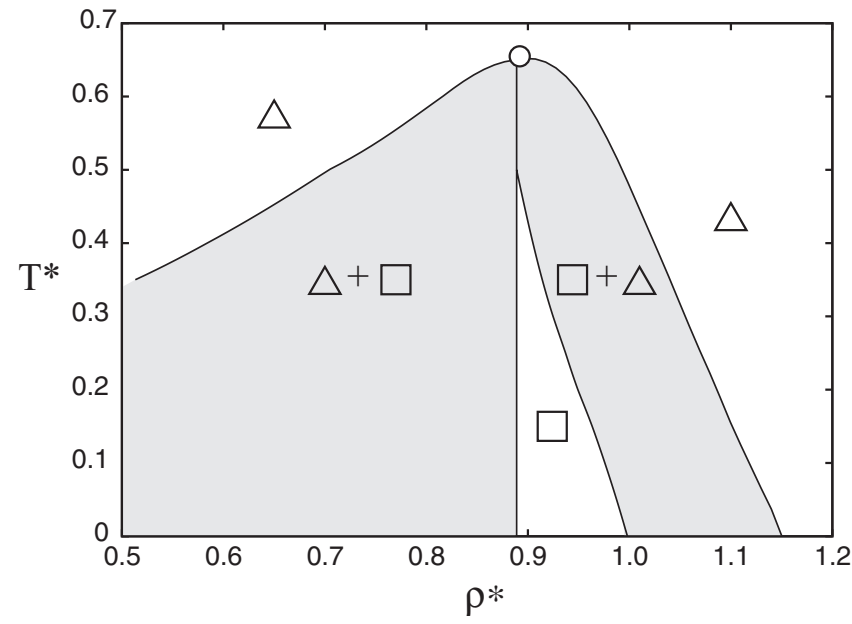

FIG. 2. Solid-solid phase diagram obtained from the mean-field analysis, in the reduced temperature $T^{*}$ vs. reduced density $\rho^{*}$ plane. Stability regions of the square and triangular solids are indicated by corresponding symbols, $\square$ and $\Delta$, respectively. Shaded regions are two-phase regions where the two coexisting phases are indicated by symbols. Big circle corresponds to the point at temperature $T_{u}$ where the first-order $\square-\triangle$ transition occurs without density change.

The phase equilibrium between the two solids is shown in Fig. 2 (note that no attempt was made to obtain the fluid part of the phase diagram due to the inaccurate character of classical mean-field theory for fluids). We find a $\Delta$ solid at low and high densities below some temperature $T_{u}$, but there is an island of $\square$ stability in between. This is because at those intermediate densities the next-nearest neighbours of the square lattice can take advantage of the attractive energy and lower the internal energy; this decrease in internal energy more than compensates for the decrease of entropy involved in going from a $\triangle$ to a $\square$ solid of the same density. At densities higher than the close-packing density of the $\square$ solid, the system turns into a $\triangle$ solid again. At high temperature the decrease in entropy associated with the $\square$ phase is too important and cannot overcome the energy term. Note that at $T_{u}$ the two structures coexist at the same density; this peculiar point has already observed in other systems ${ }^{46}$ but it is nothing but a standard first-order transition between two phases with different symmetry.

This simple theory can explain the solid region of the phase diagram qualitatively. Note that the $T=0$ is exact by construction. At high enough temperatures the approximation gives excellent results. ${ }^{46}$ At intermediate temperatures one would expect the energy approximation to be reasonable, but not the entropy since it is a geometric problem determined mainly by the density. We expect the $T=0$ phase diagram to be extended to temperatures higher than in reality, due to the underestimation of entropy.

MC and DMD simulations in the $N V T$ and $N p T$ ensembles have been used to obtain more quantitative data. The phase boundaries for the $\square-\triangle$ coexistences were obtained first by spinodal separation of the phases and in a second step, direct free-energy calculations were performed to obtain the phase boundaries. All of these results agree well with the scenario obtained from $T=0$ and mean-field theory: the $\square$ phase lives in a limited island of stability below some upper temper- ature $T_{u}$. It coexists with a lower density $\triangle$ phase and also with a higher density $\triangle$ phase and, at a special point at $T_{u}$, the two $\triangle$ phases become the same and coexist with the $\square$ phase at the same density.

Initially $N V T$-ensemble DMD simulations were performed along the isochores $\rho^{*}=0.80,0.85$, and 0.90 using $N$ $=400$ particles in a box with aspect ratio $r_{b o x}=2$. For the isochore at $\rho^{*}=0.80$ the temperature was varied in the intervals $0.18 \leq T^{*} \leq 0.53$ in steps $\Delta T^{*}=0.05$ and $0.4 \leq T^{*} \leq 0.47$ in steps $\Delta T^{*}=0.01$. For the isochore at $\rho^{*}=0.85$ two temperature intervals were used: $0.2 \leq T^{*} \leq 0.55$ in steps $\Delta T^{*}$ $=0.05$ and $0.2 \leq T^{*} \leq 0.34$ in steps $\Delta T^{*}=0.02$. For the isochore at $\rho^{*}=0.9$ the temperature interval used was: $0.2 \leq T^{*}$ $\leq 0.41$ in steps $\Delta T^{*}=0.03$. In order to check for size effects and to try a different simulation method, $N V T$-ensemble $\mathrm{MC}$ simulations were performed along the isochores $\rho^{*}=0.80$, 0.85 , and 0.90 , varying the temperature in the interval 0.23 $\leq T^{*} \leq 0.33$ in steps $\Delta T^{*}=0.01$, and using $N=4096$ particles in a box with aspect ratio $r_{b o x}=2$. A spinodal decomposition process of phase separation is present, with an interface developed in each case, indicating a typical first-order phase transition between the lower density $\triangle$ phase and the $\square$ phase. The densities of the coexistence phases were determined directly from density profiles and should be very close to the actual equilibrium coexistence values. Representative configurations where the actual separation of phases can be seen directly are presented in Fig. 3. In fact, the top panel of Fig. 3 corresponds to the coexistence between a vapor phase and the solid $\square$ phase, demonstrating the existence of a temperature below which the $\square$ phase coexists with the vapor, and above which coexistence is with a disordered $\triangle$ phase.
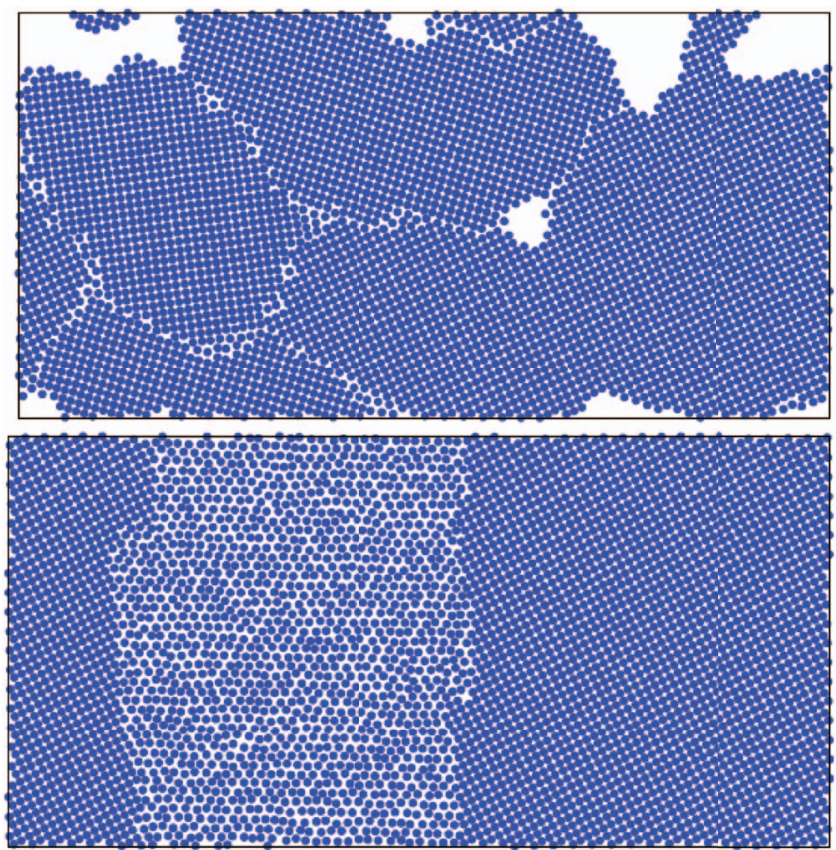

FIG. 3. Snapshots from $N V T$ simulations in the region where the square and triangle phases coexist. Box aspect ratio is $r=2$ and $N=4096$. (Upper panel) Coexistence of vapor and square solid phase at $T^{*}=0.20$, and (lower panel) coexistence of triangle and square phases for $T^{*}=0.32$. 
The coexistence of the phase with a high-density $\Delta$ phase has also been explored by generating isochores for densities $0.93 \leq \rho^{*} \leq 1.02$ with $\Delta \rho^{*}=0.01$, and for temperature $T^{*}=0.28,0.30,0.32$, and 0.33 . In this case the number of particles is $N=4096$, again with box aspect ratio $r_{b o x}=2$. The direct coexistence between the two solid phases is also observed; in this case coexistence with the vapor phase never occurs.

To obtain complementary information about the whole region where the solid-solid transitions are located, a family of isobars were simulated by MC using the $N p T$ ensemble. The exploration involves two kinds of processes, namely, warming-up and cooling-down processes at fixed pressure.

Warming-up processes were performed along several isobars, $0.5 \leq p^{*} \leq 6$, with steps $\Delta p^{*}=0.5$. Along each isobar, temperature was increased from $T^{*}=0.01$ to 0.35 in steps of $\Delta T^{*}=0.01$. For all processes, the initial configuration was a perfect triangular lattice with box ratio $r_{b o x}=2 / \sqrt{3}$. The number of particles was $N=400$.

Fig. 4 shows the isobars. The sharp density jumps can be taken as an indication of a first-order transition, and the irregular behaviour is typical of fluctuations in a two-phase region. Note that the minimum value of pressure studied is too high for the density to get below $\rho^{*} \simeq 0.9$; therefore, the stability region of the low-density $\triangle$ phase is not reached, except maybe at the lowest pressure isobars. Note that in the $N p T$ simulations to be discussed in the following, the square solid always appears at densities $\rho^{*}>0.9$. Also, note that meanfield theory predicts that the density of the square solid never decreases below $\rho^{*} \simeq 0.9$ in the entire temperature range.

To explore the low-density region and search for the lowdensity $\triangle$ to transition, cooling-down processes were generated along isobars with $0.1 \leq p^{*} \leq 5$ with step $\Delta p^{*}=0.2$. The temperature of the system was decreased, starting from $T^{*}=0.4$, to 0.25 with $\Delta T^{*}=0.01$. The initial configuration for all these simulations was a perfect triangular lattice. The box lengths were changed independently and the initial aspect ratio was $r_{b o x}=2 / \sqrt{3}$. The number of particles was $N$ $=400$. Results are contained in Fig. 5. In most of the simulations, the isobars show an abrupt change corresponding to a

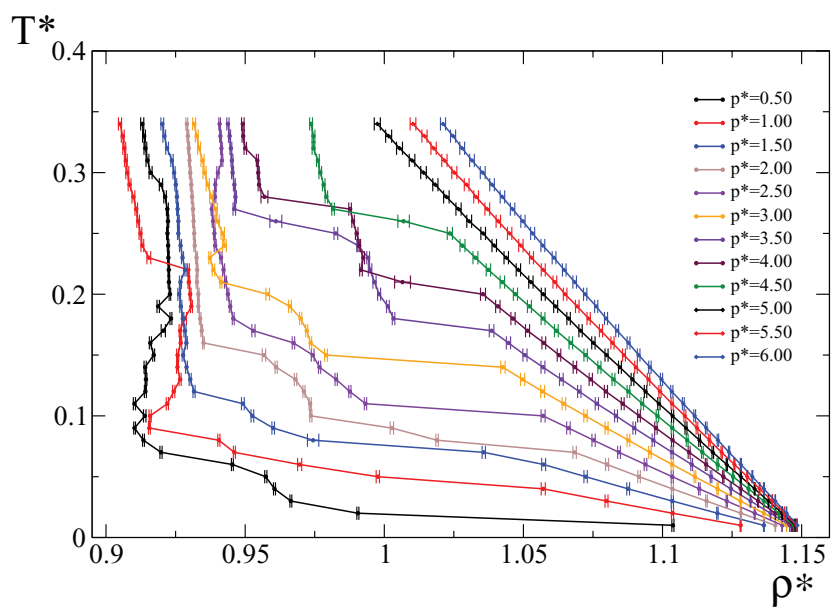

FIG. 4. Warming processes along isobars $p^{*}=0.5-6.0$ with increments of $\Delta p^{*}=0.5$.

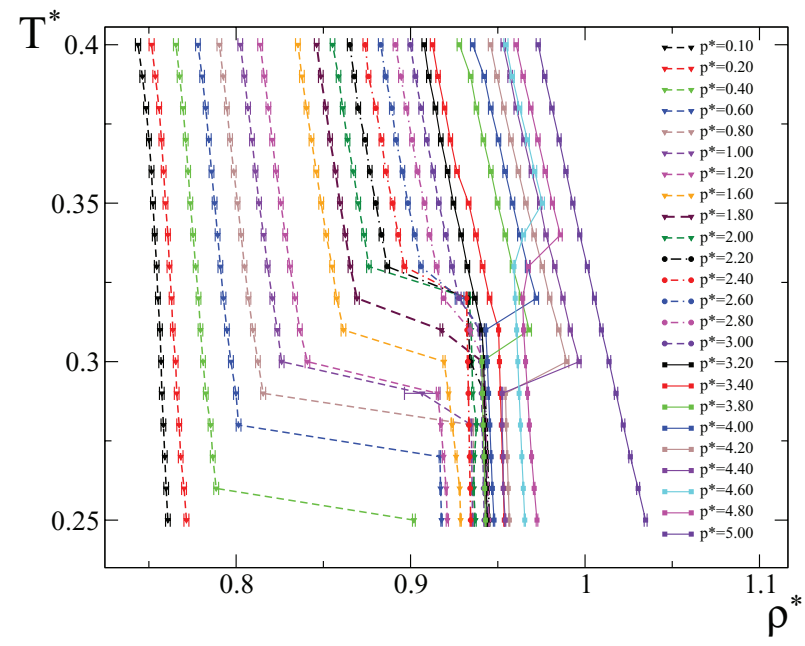

FIG. 5. Cooling down processes along isobars with $p^{*}=0.1-5.0$.

visible jump in the system structure. For the pressure interval $0.4 \leq p^{*} \leq 3$, as the system cools down and the temperature reaches a particular value that depends on the particular isobar, the structure of the system suffers a sudden change from the $\Delta$ to the solid, which can be inferred directly from particle configurations. The final point of the isobars falls approximately within the small density interval where the mean-field theory predicts the phase to be stable. For higher values of pressure, $3.8 \leq p^{*} \leq 4.8$, the isobars show a jump going from the high-density $\Delta$ to the solid. In this case the final points of the isobars also fall close to that small density interval and the structural change from $\Delta$ solid of low density to the solid is corroborated by the particle snapshots.

\section{B. Liquid-solid phase boundary}

For many years, the study of melting in 2D dimensions has generated much controversy, one of the reasons being that the results obtained from the numerical simulations are strongly influenced by system size. This has led to the publication of several simulation studies that are not mutually consistent. Some of them suggest that the transition occurs in a single step and as a first-order transition, as in the 3D case. Other results show that the transition is performed in two steps: from a solid to an intermediate region and from it to the liquid phase. The first step, starting from a 2D solid characterized by quasi-long range positional correlations $G_{T}$ and long-range bond orientational correlation $G_{6}$, involves melting to an intermediate phase called hexatic phase, characterized by quasi-long range bond orientational order. The second step proceeds from the hexatic to the liquid phase, where both $G_{6}$ and $G_{T}$ are short ranged. This mechanism is consistent with that predicted by KTHNY theory, ${ }^{14,15}$ which considers the two phase transitions to be continuous. However, recent studies have found evidence that the solid-hexatic transition is continuous, while the hexatic-liquid transition is of first order. ${ }^{47}$ To investigate the existence of the hexatic phase in our case, the local bond orientational order parameter $\Psi_{6}$, its configurational average $\Psi_{6}$, and its correlation function $G_{6}$ were obtained. 

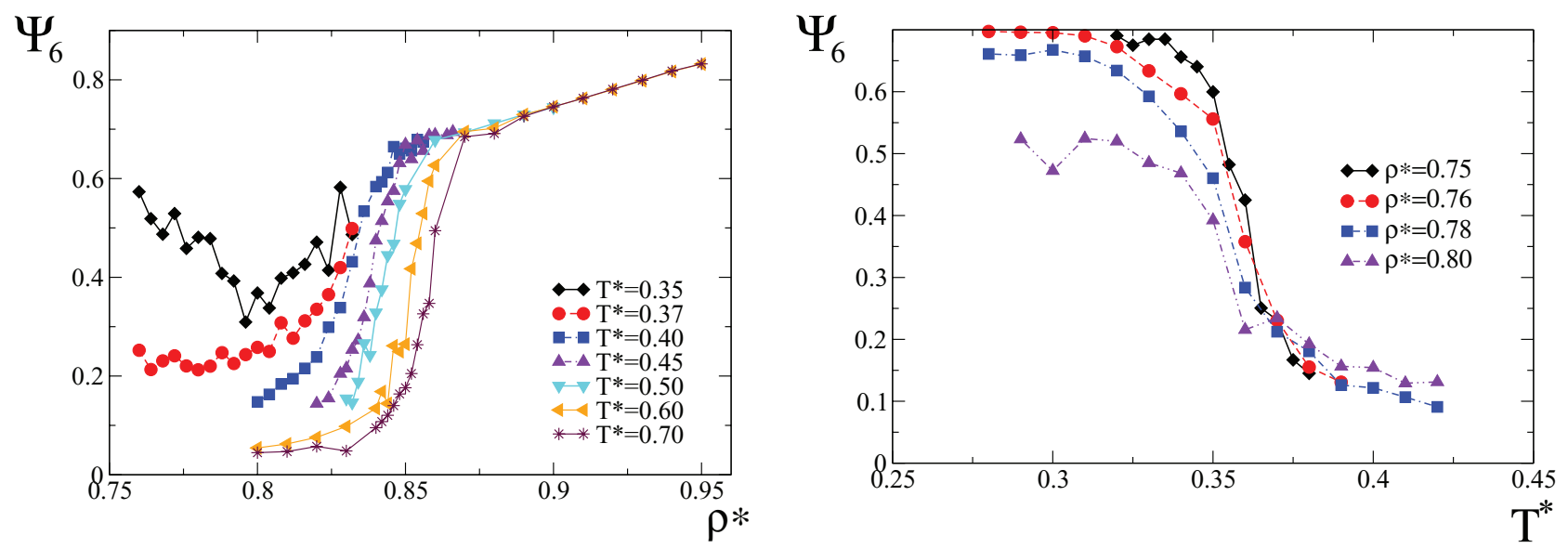

FIG. 6. Orientational order parameter as a function of (a) density and for different temperature, and (b) temperature and for different densities.

In Fig. 6(a) the order parameter $\Psi_{6}=\Psi_{6}\left(\rho^{*}\right)$ is presented along the isotherms $T^{*}=0.35,0.37,0.4,0.45,0.5$, 0.6 , and 0.7 , using systems with $N=4096$ particles. For $\rho^{*}$ $=0.8$, the order parameter is quite small, $\Psi_{6} \simeq 0.1$, while for $\rho^{*}=0.875$ it increases to $\simeq 0.7$. This suggests that for these isotherms the liquid-hexatic transition density is in the range $0.82<\rho^{*}<0.87$. Complementary information is also provided from the temperature dependence $\Psi_{6}=\Psi_{6}\left(T^{*}\right)$ for the isochores $\rho^{*}=0.75,0.76,0.78$, and 0.8 . This is presented in Fig. 6(b). We see, for example, that for $T^{*}=0.3$ the order parameter is relatively large, $\Psi_{6}>0.5$, while for $T^{*}=0.37$, we have $\Psi_{6}<0.2$ in all cases. This seems to indicate that the system is in a hexatic phase for $T^{*}=0.35$ in the density range $0.75<\rho^{*}<0.80$.
The bond orientational correlation function was also computed for a family of isochores for temperatures $T^{*}$ $=0.35,0.37,0.40,045,0.50,0.60$, and 0.70. Some of them are shown in Fig. 7. Taking into account the criterion of quasi-long-range decay with a $r^{-1 / 4}$ law for the liquid-tohexatic transition, a continuous transition results for densities $\rho^{*}>0.84$.

A different route to verify our results is to evaluate the Binder cumulant, ${ }^{44} U_{L}^{4}$. In this case the calculation was based on a system-size analysis as a function of the density: the simulation box was subdivided in 13 subsystems of different size. The results, for the same temperatures at which $G_{6}$ was evaluated, are shown in Fig. 8. Direct inspection of the figure indicates that, for each temperature and within the accuracy of
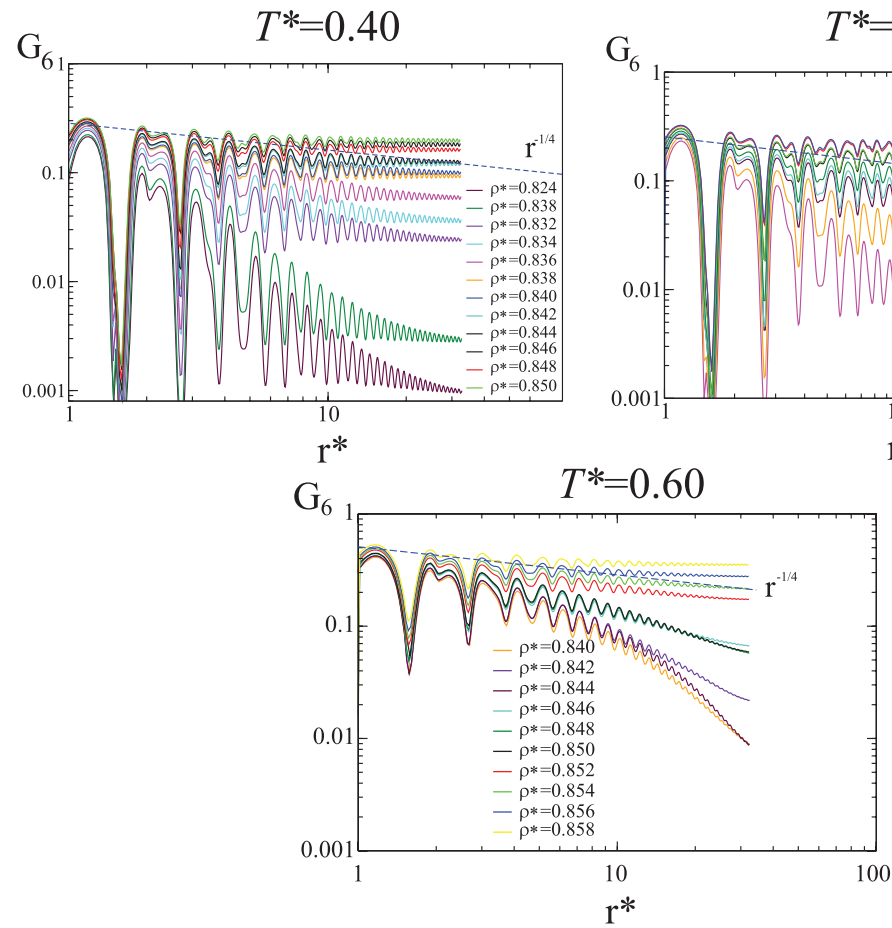
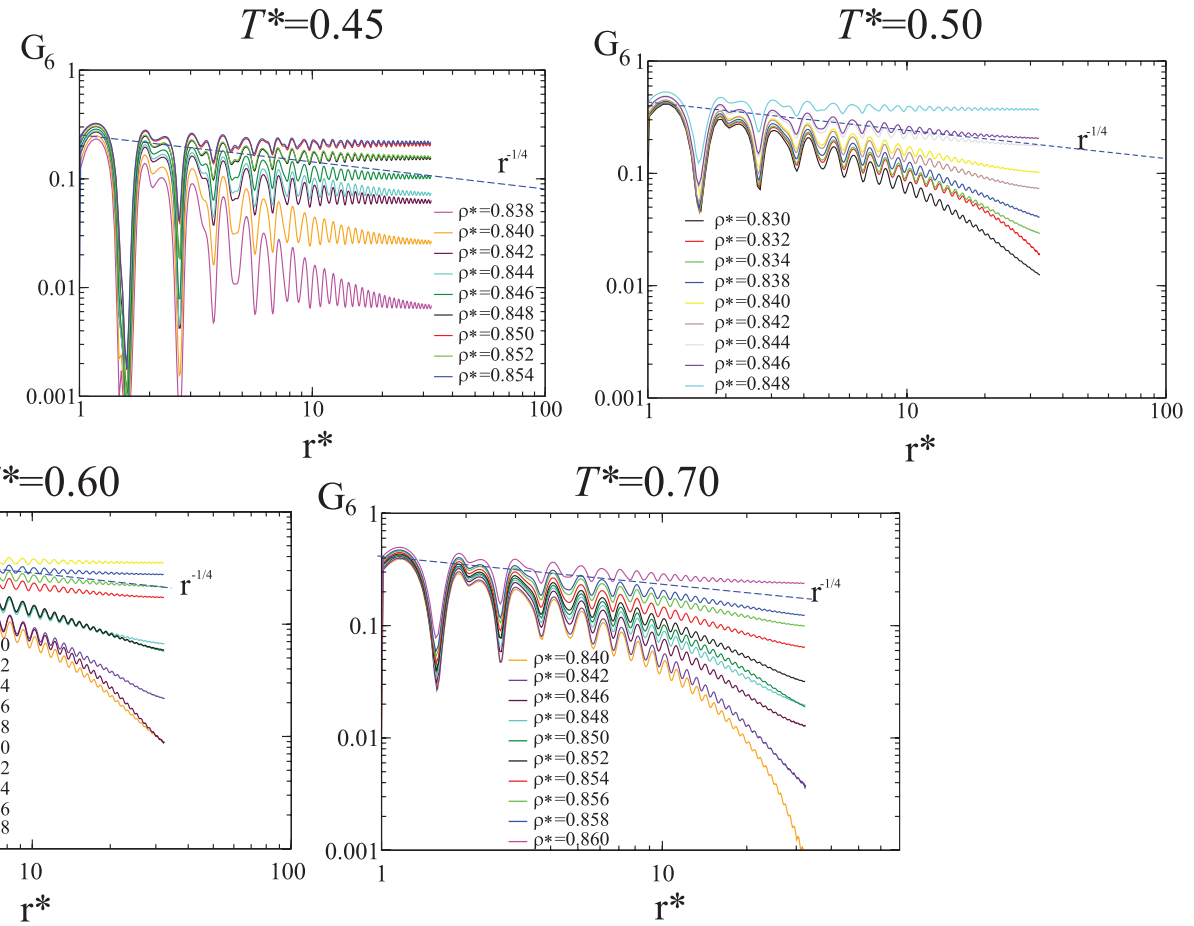

FIG. 7. Orientational correlation function, in a $\log \log$ scale, for $T^{*}=0.40,0.45,0.5,0.6,0.7$. Densities are indicated in the corresponding insets. 
$\mathrm{U}^{4}$

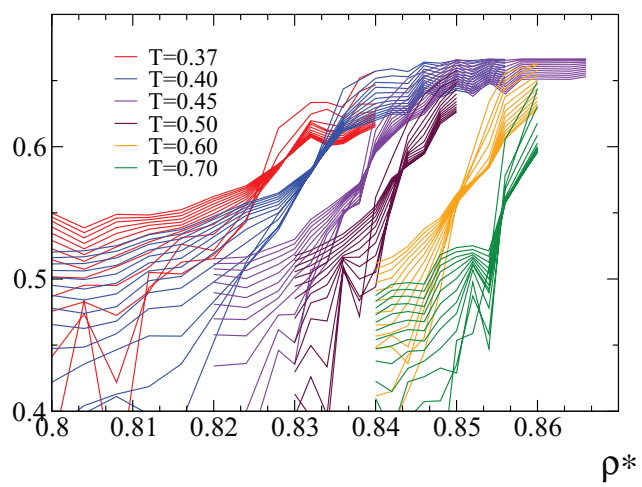

FIG. 8. Fourth-order Binder cumulant of the bond orientational order as a function of the density at temperatures $T^{*}=0.37,0.40,0.45,0.50,0.60$, and 0.70

the data, all curves collapse to a single point, which means that the liquid-hexatic transition is of first order (otherwise the hexatic phase would only at a fixed density, which is not possible thermodynamically). This result is in agreement with the most recent and deeper analysis of the hard-disc system; ${ }^{47}$ note that, usually, attractive interactions reinforce the order of first-order transitions. The densities where the cumulants intersect, at the different temperatures, are similar to those obtained using a criterion based on the $\Psi_{6}$ order parameter.

\section{Complete phase diagram}

To confirm and determine in a more quantitative way the solid-solid transitions obtained with the previous $N V T$ and $N p T$ simulations, we have used a version ${ }^{48}$ of the well-known Frenkel and Ladd method ${ }^{37}$ to obtain the absolute free energy of the solid phases. The method is based on the knowledge of the free energy of a reference system which is coupled to the actual system via thermodynamic integration; this gives the change of free energy between the reference and actual systems. Values of the integrand are obtained by the corresponding NVT MC simulation. In our case we performed 10 MC simulations and used the Gauss-Legendre quadrature to evaluate the thermodynamic integral. The numbers of particles used in these simulations were $N=882$ for the $\triangle$ solid and $N=900$ for the $\square$ solid. We checked that the size effects were negligible. The problem with the centre-of-mass constraint $^{49}$ is also negligible for the system sizes used. The number of the MC steps used was $10^{4}$ for equilibration, while for averaging $2 \times 10^{4}$ steps were used. The Maxwell construction was used to obtain the different solid-solid transitions. The results are shown in Fig. 9. They confirm the previous $N V T$ and NpT analyses. Note the qualitative agreement with mean-field predictions. As expected, the value for the $T_{u}$ temperature is lower than that predicted by mean-field theory.

Fig. 9 contains all relevant data obtained in the present work. In particular, from the analysis of the liquid-to-hexatic transition presented in Sec. III B, we only can conclude that the liquid-hexatic transition at $T^{*}=0.40$ must be at a density $\rho^{*} \simeq 0.82$ and that the transition density increases slightly with the temperature, as is shown in the phase diagram. The

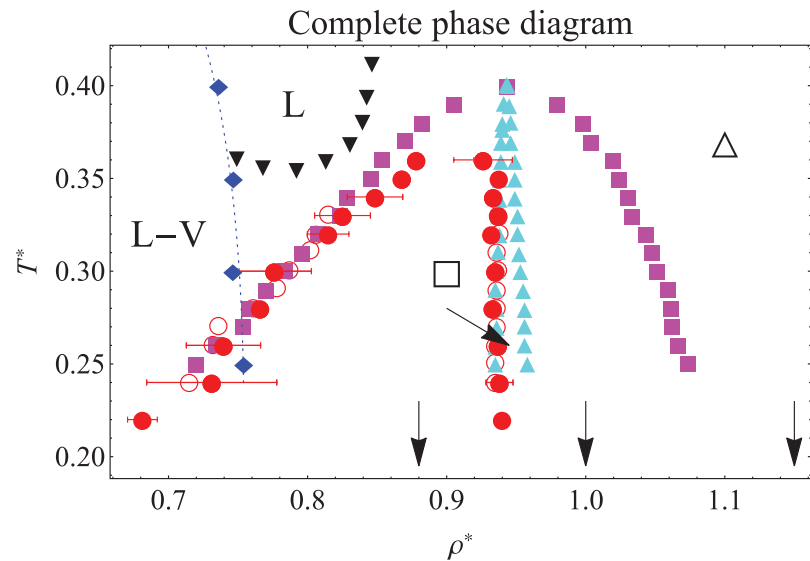

FIG. 9. Phase diagram in the $T^{*}-\rho^{*}$ plane as obtained from computer simulation. Diamonds correspond to the liquid branch of the liquid-vapor phase transition, taken from Ref. 49. Solid-solid transitions: open circles from $N V T$ simulations; full circles from $N V T$ DMD simulations; solid squares and triangles from free-energy thermodynamic integration calculations. Inverted triangles: liquid-hexatic transition. Thick arrows indicate coexistence densities at $T=0$.

unusual behavior found for the liquid-hexatic transition at lower temperature is also shown. In the figure we have drawn a dotted curve that connects both branches of the transition. However, other scenarios could be possible. Finally, we have tried to obtain information on the hexatic-to-triangular solid transition using the translational order parameter and correlation function. Within the accuracy of our limited-size systems, we have been unable to extract any useful conclusion as to the location and nature of the transition.

\section{CONCLUSIONS}

The phase diagram of a two-dimensional square-well model with a range parameter $\lambda=1.5 \sigma$ has been investigated using a variety of techniques: analysis at $T=0$, mean-field theory, canonical and isothermal-isobaric MC, and canonical DMD. In the ground state a solid phase with a square symmetry is stabilised within a small density interval. This solid coexists with a zero-density vapor at the left side of the interval and with a perfect triangular solid at the right side. A simple mean-field calculation shows that the density interval where the square solid is stable shrinks with temperature, and disappears at a temperature $T_{u}$ where the square solid coexists with a triangular solid of the same density. The simple theory was useful to identify the possible solid phases. Computer simulations corroborated this scenario while at the same time allowed us to expand the analysis to the rest of the phase diagram.

Systems with different numbers of particles were employed in the simulations; the larger ones, $N=65536$, were used to study the region between liquid and solid, where indications of a hexatic phase were found. We obtained information arising from several properties: translational and orientational order parameters and their corresponding correlation functions, equations of state, density profiles, and visual inspection from the snapshots with different box shapes and number of particles. Also, free-energy calculations were 
performed to study the solid region. All this information, together with that from our recent work on the liquid-vapor phase equilibrium of the same fluid, ${ }^{49}$ allow us to obtain a complete phase diagram including vapor, liquid, and the two solid phases with square $\square$ and triangular $\Delta$ symmetry. Also, indications for a hexatic phase between the liquid and the solid phase were obtained in the present work. An approximate location for the liquid-to-hexatic transition was obtained. Our evidence suggests that the transition is of first order.

A complete phase diagram was drawn by gathering all of the above results. A square solid was found to be stabilized below a reduced temperature $T^{*} \simeq(0.39-0.40)$ in a narrow density window. This solid stabilizes below its closepacking density at densities such that the next-nearest neighbours can take advantage of the attractive well and this extra energy more than compensates the reduction in entropy with respect to the $\Delta$ solid. The phase coexists with the $\Delta$ phase along low- and high-density branches at temperatures below $T^{*} \simeq(0.39-0.40)$; at this point both phases coexist at the same density, $\rho^{*} \simeq 0.943$. There appears to be a triple point at $T^{*} \simeq 0.27$ and $\rho \simeq 0.750$ involving vapor and triangular $\Delta$ and square $\square$ solids. Even though enough evidence is presented to validate the existence of a hexatic phase in the region $0.27<T^{*}<0.40$, and even though a large system (50 000 particles) was simulated, the question of the influence of the finite size is not fully addressed so whether or not the triangular solid is in fact a hexatic phase is open to question.

\section{ACKNOWLEDGMENTS}

The authors acknowledge the following financial support: "Investigación realizada gracias al Programa UNAMDGAPA-PAPIIT IN104111-2 Transiciones de fases y agregados moleculares en 2 dimensiones;" CONACYT grant Efecto de la quiralidad, polaridad y anisotropía en el auto-ensamblaje molecular en dos dimensiones. J.C.A.-P. is grateful for a scholarship from CONACYT. We acknowledge financial support from programme MODELICOCM/S2009ESP-1691 (Comunidad Autónoma de Madrid, Spain), and FIS2010-22047-C01 and FIS2010-22047-C04 (MINECO, Spain).

${ }^{1}$ M. Lisal and I. Nezbeda, Mol. Phys. 96, 335 (1999); Y. Y. Duan, L. Shi, L. Q. Sun, M. S. Zhu, and L. Z. Han, Int. J. Thermophys. 21, 393, (2000)

${ }^{2}$ C. J. Peng, H. L. Liu, and Y. Hu, Chin. J. Chem. 19, 1165 (2010).

${ }^{3}$ K. Rah and B. C. Eu, J. Chem. Phys. 116, 7967 (2002).

${ }^{4}$ A. Skibinsky, S. V. Buldyrev, A. Scala, S. Havlin, and H. E. Stanley, Phys. Rev. E 60, 2664 (1999).

${ }^{5}$ E. Zaccarelli, G. Foffi, K. A. Dawson, F. Sciortino, and P. Tartaglia, Phys. Rev. E 63, 031501 (2001); W. Gotze and M. Sperl, J. Phys.: Condens. Matter 15, S869 (2003).

${ }^{6}$ L. A. Del Pino, A. L. Benavides, and A. Gil-Villegas, Mol. Simul. 29, 345 (2003); S. Q. Zhou, J. Phys. Chem. B 107, 3585 (2003).

${ }^{7}$ K. Shukla and R. Rajagopalan, Mol. Phys. 81, 1093 (1994).

${ }^{8}$ B. H. Chang, and Y. C. Bae, Biomacromolecules 4, 1713 (2003); J. J. Grigsby, H. W. Blanch, and J. M. Prausnitz, Biophys. Chem. 91, 231 (2001); K. Murakami, Langmuir 15, 4270 (1999).
${ }^{9}$ S. Saadeddine, J. F. Wax, B. Grosdidier, J. G. Gasser, C. Regnaut, and J. M. Dubois, Phys. Chem. Liq. 28, 221 (1994); J. N. Nzali and W. Hoyer, Z. Naturforsch. A: Phys. Sci. 55a, 381 (2000).

${ }^{10}$ G. T. Evans, J. Chem. Phys. 115, 1440 (2001); B. Cernuschi-Frias, H. J. Gonzalez, and F. Cernuschi, Mol. Phys. 94, 505 (1998).

${ }^{11}$ P. Bolhuis, M. Hagen, and D. Frenkel, Phys. Rev. E 50, 4880 (1994).

${ }^{12}$ D. A. Young and B. J. Alder, J. Chem. Phys. 73, 2430 (1980).

${ }^{13}$ J. Serrano-Illán, G. Navascués, and E. Velasco, Phys. Rev. E 73, 011110 (2006).

${ }^{14}$ J. M. Kosterlitz and D. J. Thouless, J. Phys. C: Solid State Phys. 6, 1181 (1973).

${ }^{15}$ D. R. Nelson, Defects and Geometry in Condensed Matter Physics (Cambridge University Press, 2002).

${ }^{16}$ P. Nassoy, M. Goldmann, O. Bouloussa, and F. Rondelez, Phys. Rev. Lett. 75, 457 (1995); T. Huang, Z. P. Hu, A. D. Zhao, H. Q. Wang, B. Wang, J. L. Yang, and J. G. Hou, J. Am. Chem. Soc. 129, 3857 (2007).

${ }^{17}$ A. K. Geim and K. S. Novoselov, Nat. Mater. 6, 183 (2007).

${ }^{18}$ D. Henderson, O. H. Scalise, and W. R. Smith, J. Chem. Phys. 72(4), 2431 (1980).

${ }^{19}$ H. S. Gulati and C. K. Hall, J. Chem. Phys. 108, 7478 (1998).

${ }^{20}$ G. Orkoulas and A. Z. Panagiotopoulos, J. Chem. Phys. 110, 1581 (1999).

${ }^{21}$ M. G. Noro and D. Frenkel, J. Chem. Phys. 113, 2941 (2000).

${ }^{22}$ F. del Río, E. Ávalos, R. Espíndola, L. F. Rull, G. Jackson, and S. Lago, Mol. Phys. 100(15), 2531 (2002).

${ }^{23}$ G. J. Gloor, G. Jackson, F. J. Blas, and E. de Miguel, J. Chem. Phys. 123, 134703 (2005).

${ }^{24}$ J. R. Errington and D. A. Kofke, J. Chem. Phys. 127, 174709 (2007).

${ }^{25}$ W. R. Smith, D. Henderson, and Y. Tago, J. Chem. Phys. 67, 5308 (1977).

${ }^{26}$ W. G. Chapman, G. Jackson, K. E. Gubbins, and M. Radosz, Fluid Phase Equilib. 52, 31 (1989).

${ }^{27}$ R. Espíndola-Heredia, F. Del Río, and A. Malijevsky, J. Chem. Phys. 130, 024509 (2009).

${ }^{28}$ C. McCabe, A. Gil-Villegas, G. Jackson, and F. del Rio, Mol. Phys. 97, 551 (1999).

${ }^{29}$ G. J. Pauschenwein and G. Kahl, J. Chem. Phys. 129, 174107 (2008).

${ }^{30}$ W. H. Zurek and W. C. Shieve, Phase Transitions 2, 31 (1981).

${ }^{31}$ F. del Río and A. GilVillegas , J. Phys. Chem. 95, 787 (1991).

${ }^{32}$ H. L. Vortler, K. Schafer, and W. R. Smith, J. Phys. Chem. B 112, 4656 (2008); H. L. Vortler, Collect. Czech. Chem. Commun. 73, 518 (2008).

${ }^{33}$ G. T. Gao, X. C. Zeng, and W. Wang, J. Chem. Phys. 106(8), 3311 (1997).

${ }^{34}$ J. Wang, M. Chen, and Z. Guo, Chin. Sci. Bull. 48(7), 623 (2003).

${ }^{35}$ P. Bladon and D. Frenkel, Phys. Rev. Lett. 74, 2519 (1995).

${ }^{36}$ P. Allen and D. J. Tildesley, Computer Simulations of Liquids (Oxford University Press, New York, 1987).

${ }^{37}$ D. Frenkel and B. Smith, Understanding Molecular Simulation from Algorithms to Applications Second Edition (Academic Press, San Diego, 2002).

${ }^{38}$ B. J. Alder and T. E. Wainwright, J. Chem. Phys. 31, 459 (1959).

${ }^{39}$ D. C. Rapaport, J. Chem. Phys. 71, 3299 (1979).

${ }^{40}$ G. A. Chapela, S. E. MartínezCasas, and J. Alejandre, Mol. Phys. 53, 139 (1984).

${ }^{41}$ P. M. Chaikin and T. C. Lubensky, Principles of Condensed Matter Physics (Cambridge University Press, 2000).

${ }^{42}$ P. Dillmann, G. Maret, and P. Keim, J. Phys.: Condens. Matter 24, 464118 (2012).

${ }^{43}$ M. de Berg, M. van Kreveld, M. Overmars, and O. Schwarzkopf, Computational Geometry: Algorithms and Applications (Springer, 2000).

${ }^{44}$ H. Weber, D. Marx, and K. Binder, Phys. Rev. B 51, 14636 (1995).

${ }^{45}$ E. Velasco, L. Mederos, and G. Navascués, Mol. Phys. 97, 1273 (1999).

${ }^{46}$ E. Velasco, L. Mederos, G. Navascués, P. C. Hemmer, and G. Stell, Phys. Rev Lett. 85, 122 (2000).

${ }^{47}$ E. P. Bernard and W. Krauth, Phys. Rev Lett. 107, 155704 (2011).

${ }^{48}$ The basic difference between the original method of Frenkel and Ladd and ours is the potential used to couple the reference system with the actual system; details of this version will be published elsewhere. We have checked that our results are numerically identical to those obtained with the Frenkel and Ladd method.

${ }^{49}$ J. C. Armas-Pérez, J. Quintana-H, and G. A. Chapela, J. Chem. Phys. 138, 044508 (2013). 